\title{
Audit of nutritional provision in a high dependency unit
}

\author{
K. Morrison \\ Department of Nutrition and Dietetics, Falkirk Royal Infirmary, Major's Loan, Falkirk FK1 5QE, UK
}

Up to $60 \%$ of patients in UK hospitals are clinically malnourished ${ }^{(1)}$. Some of these patients are admitted to the high-dependency unit (HDU) post surgery, and research shows that early post-operative nutrition reduces infection rates and shortens the length of stay in hospital $^{(2)}$.

In order to review the nutritional provision to patients in the combined medical and surgical HDU in Forth Valley a data collection form was developed. The HDU had no formal nutrition protocols or screening and it was suspected that many patients might have inadequate intake during their stay.

All patients admitted to HDU during a 1-month period were considered eligible, but only those who stayed $>48 \mathrm{~h}$ were included. Data were collected Monday-Friday by the unit dietitian and retrospectively for weekends. Data collection included form of intake (if any), weight, diagnosis, risk of refeeding syndrome and nutritional-associated complications. Actual energy intake for parenteral and enteral nutrition was calculated, allowing any energy deficit to be calculated.

The average stay on HDU was $7 \mathrm{~d}$. Details for thirty-two patients were collected (71\% surgical; $28 \%$ medical). A total of $21 \%$ ( $n$ 5) of the surgical patients were parenterally-fed and $26 \%(n 6)$ tube-fed. All feeds except one were started in the intensive care unit before transfer to the HDU. In total $56 \%$ ( $n$ 13) were indicated to have an inadequate dietary intake and were not otherwise fed. Less than half these patients were referred to the dietitian. No medical patients required parenteral or tube feeds but $88 \%(n 8)$ were indicated to have an inadequate dietary intake with $62 \%(n 5)$ of those being referred to the Dietitian. The average energy deficit on leaving HDU was $61 \%$ for patients solely receiving enteral or parenteral nutrition. Two patients remained 'nil by mouth' for their entire stay on the HDU. Four patients in total were thought to be at risk of refeeding syndrome due to a period of 'nil by mouth' or very poor intake while in hospital ${ }^{(3)}$.

The provision of nutrition on HDU in Forth Valley was found to be variable and could be improved to avoid long periods in which no nutrition is provided. This finding has led to the development of protocols for starting enteral feeding, and guidelines for enteral and parenteral feeding and nutritional screening have been introduced to HDU.

1. Ruxton CHS, Gordon J, Kirkwood L et al. (2008) J Hum Nutr Diet 21, 81-90.

2. Lewis SJ, Egger M, Sylvester PA et al. (2001) Brit Med J 323, 773-776.

3. National Institute for Health and Clinical Excellence (2006) Nutrition Support in Adults: Oral Nutrition Support, Enteral Tube Feeding and Parenteral Nutrition. Clinical Guideline 32. London: NICE. 